\title{
Blood and Brain Transcriptome Analysis Reveals APOE Genotype-mediated and Immune-related Pathways Involved in Alzheimer Disease
}

\section{Rebecca Panitch}

Boston University School of Medicine

Junming $\mathrm{Hu}$

Boston University School of Medicine

Weiming Xia

Boston University School of Medicine

David A Bennett

Rush University Medical Center

Thor D Stein

Boston University School of Medicine

\section{Lindsay A Farrer}

Boston University School of Medicine

Gyungah R. Jun ( $\nabla$ gyungah@bu.edu )

Boston University School of Medicine https://orcid.org/0000-0002-3230-8697

\section{Research Article}

Keywords: Alzheimer's disease, blood brain barrier, APOE, differential expression, co-expression network, vascular injury

Posted Date: September 17th, 2021

DOI: https://doi.org/10.21203/rs.3.rs-892590/v1

License: (c) (1) This work is licensed under a Creative Commons Attribution 4.0 International License. Read Full License

Version of Record: A version of this preprint was published at Alzheimer's Research and Therapy on February 9th, 2022. See the published version at https://doi.org/10.1186/s13195-022-00975-z. 
Blood and Brain Transcriptome Analysis Reveals $A P O E$ Genotype-mediated and Immunerelated Pathways Involved in Alzheimer Disease

Rebecca Panitch ${ }^{1}$, Junming $\mathrm{Hu}^{1}$, Weiming Xia ${ }^{2,6}$, David A. Bennett ${ }^{7}$, Thor D. Stein ${ }^{3,6}$, Lindsay A. Farrer $^{1,4,5,8,9}$, Gyungah R. Jun ${ }^{1,5,8, *}$

Departments of ${ }^{1}$ Medicine (Biomedical Genetics), ${ }^{2}$ Pharmacology \& Experimental Therapeutics, ${ }^{3}$ Pathology \& Laboratory Medicine, ${ }^{4}$ Neurology, and ${ }^{5}$ Ophthalmology, Boston University School of Medicine, 72 East Concord Street, Boston, MA 02118, USA.

${ }^{6}$ Department of Veterans Affairs Medical Center, Bedford, MA 01730, USA.

${ }^{7}$ Rush Alzheimer's Disease Center, Rush University Medical Center, 1750 W. Harrison Street, Suite 1000, Chicago, IL 60612, USA.

Departments of ${ }^{8}$ Biostatistics and ${ }^{9}$ Epidemiology, Boston University School of Public Health, 715 Albany Street, Boston, MA 02118, USA.

* Address Correspondence to:

Gyungah R. Jun, PhD, Department of Medicine (Biomedical Genetics), Boston University School of Medicine, 72 East Concord Street, Boston, MA 02118, USA 02118, email: gyungah@bu.edu. 


\begin{abstract}
Background: While Alzheimer disease (AD) is generally considered as a brain disorder, blood biomarkers may be useful for diagnosis and prediction of AD brain pathology. The APOE $\varepsilon 4$ allele has shown cerebrovascular effects including acceleration of blood brain barrier breakdown.
\end{abstract}

Methods: We evaluated differential expression of previously established AD genes in brains from 344 pathologically confirmed AD cases and 232 controls and in blood from 112 pathologically confirmed AD cases and 67 controls from the Religious Orders Study and Memory and Aging Project. Differential gene expression between AD cases and controls was analyzed in the blood and brain jointly using a multivariate approach in the total sample and within $A P O E$ genotype groups. Gene set enrichment analysis was performed within APOE genotype groups using the results from the combined blood and brain analyses to identify biologically important pathways. Gene co-expression networks in brain and blood samples were investigated using weighted correlation network analysis. Top ranked genes from networks and pathways were further evaluated with vascular injury traits.

Results: We observed differentially expressed genes with $\mathrm{P}<0.05$ in both brain and blood for established AD genes INPP5D (upregulated) and HLA-DQAI (downregulated). PIGHP1 and FRAS1 were differentially expressed at the transcriptome-wide level $\left(\mathrm{P}<3.3 \times 10^{-6}\right)$ within $\varepsilon 2 / \varepsilon 3$ and $\varepsilon 3 / \varepsilon 4$ groups, respectively. Gene-set enrichment analysis revealed 21 significant pathways (false discovery rate $\mathrm{P}<0.05)$ in at least one $A P O E$ genotype group. Ten pathways were significantly enriched in the $\varepsilon 3 / \varepsilon 4$ group, and six of these were unique to these subjects. Four pathways were enriched for $\mathrm{AD}$ upregulated genes in the $\varepsilon 3 / \varepsilon 4$ group and $\mathrm{AD}$ downregulated genes in $\varepsilon 4$ lacking subjects. We identified a co-expressed gene network in brain that reproduced in blood and showed 
higher average expression in $\varepsilon 4$ carriers. Twenty-three genes from pathway and network analyses were significantly associated at $\mathrm{P}<0.05$ with at least one vascular injury trait.

Conclusion: These results suggest that $A P O E$ genotype contributes to unique expression network profiles in both blood and brain. Several genes in these networks are associated with measures of vascular injury and potentially contribute to $\varepsilon 4$ 's effect on the blood brain barrier.

Keywords: Alzheimer's disease, blood brain barrier, APOE, differential expression, coexpression network, vascular injury 


\section{Background}

Alzheimer disease (AD) is a neurodegenerative disorder characterized by amyloid plaques and neurofibrillary tau tangles in the brain [1]. Because these hallmark proteins are sometimes detectable in blood before clinical symptoms appear, there are on-going efforts to identify bloodbased signatures from multi-omics and biomarker data that can facilitate detection of $\mathrm{AD}$ preclinically $[2,3]$.

Cerebrovascular AD-related pathology that may affect the blood brain barrier (BBB), such as cerebral amyloid angiopathy (CAA) has been shown to exacerbate neurodegeneration and neuroinflammation [4]. Dysfunction of the BBB, a semi-permeable border separating the extracellular fluid and brain tissue from circulating blood, has been implicated in the accumulation of amyloid- $\beta(\mathrm{A} \beta)$ and hyperphosphorylation of tau protein [5,6]. Apolipoprotein $\mathrm{E}(\mathrm{APOE})$ genotype is the strongest genetic risk factor for late onset $\mathrm{AD}$ and the $\varepsilon 4$ allele has been recently associated with $\mathrm{BBB}$ dysfunction leading to cognitive decline $[7,8]$. Heterozygosity of the $A P O E$ $\varepsilon 4$ allele confers 3-4 fold increase of AD risk and $\varepsilon 4$ homozygotes have a 10-12 fold increased likelihood of a clinical diagnosis of AD among persons of European ancestry [7,9]. By contrast, among clinically and neuropathologically confirmed AD cases and controls of European ancestry, a single copy of the $A P O E \varepsilon 2$ allele is associated with $61 \%$ decreased risk and $\varepsilon 2$ homozygotes have an $87 \%$ reduced risk for $\mathrm{AD}$ compared to individuals with the $\varepsilon 3 / \varepsilon 3$ genotype [10]. Cerebrovascular AD-related pathologies have also shown APOE genotype-dependent patterns. Both $\varepsilon 2$ and $\varepsilon 4$ are significantly associated with CAA [11].

Previous whole transcriptome-wide studies from autopsied brains demonstrate that the classical complement cascade and tau phosphorylation are linked to $\mathrm{AD}$ in an $A P O E$ genotypespecific manner [12,13]. However, expression profiles associated with AD has not been intensively 
investigated in blood and brain from the same individuals, especially separated by APOE genotype. Here, we analyzed gene expression measured in blood and brain tissue obtained from participants of the Religious Orders Study and Rush Memory and Aging Project (ROSMAP) [14] stratified by APOE genotype in order to discern AD-related differential gene expression, biological pathways, and gene networks shared in blood and brain.

\section{Methods}

\section{Sources of Blood and Brain Transcriptomic and Phenotypic Data}

RNA sequencing (RNA-seq) data generated from blood donated by 614 ROSMAP participants and phenotypic data collected from those subjects were obtained from the Synapse portal [15]. RNA batches were prepared using a SMART-seq2 protocol (batches 1-2) or a SMART-seq2 like protocol (batch 3). Batch 1 ( $2 \times 101 \mathrm{bp})$ and Batch 2 ( 2 x 76bp) were pooled and sequenced by HiSeq 2500 (Illumina). Batch 3 ( 2 x 50 bp) was pooled and sequenced on Nova Seq 6000 (Illumina). A post-mortem diagnosis of AD was established for 112 participants using NIAReagan criteria including Braak staging for assessing the severity of neurofibrillary tangles and the Consortium to Establish a Registry for Alzheimer Disease (CERAD) semi-quantitative measure for neuritic plaques (CERAD Score). Another 67 participants who were clinically normal showed no pathological evidence of $\mathrm{AD}$ and were included in this study as controls (Supplementary Table 1) [14,16]. Age, sex, sequencing batch, and library batch information was available for all subjects. Publicly available prefrontal cortex brain RNA-seq and neuropathological data for 639 ROSMAP participants were obtained from the Synapse portal [15]. Sequencing libraries were prepared using the strand-specific dUTP method with poly-A selection, and all samples were sequenced using an Illumina HiSeq instrument. Of these 639 samples, data 
from 576 samples with both RNA integrity number (RIN) and post-mortem interval (PMI) were included in subsequent differential expression analyses (Supplementary Table 1). Previously reported RNA-seq data were also available which were derived from the frontal cortex tissue region of 208 frontal autopsied brains (64 AD and 129 controls) donated to the Framingham Heart Study and Boston University Alzheimer's Disease Center (FHS/ADRC) [12]. A diagnosis of AD in these brains was established using NIA-Regan criteria including Braak staging and CERAD score [12].

\section{Quality Control, Mapping, and Quantification of Gene Expression Data and Sample}

The 614 FASTQ files derived from blood RNA-seq data were processed in batches. Quality control (QC) of the sequence data was performed using FastQC which checked for over-abundance of adaptors and over-represented sequences [17]. Reads passing initial QC were aligned to the human reference genome (GRCh38.95) using STAR (version 2.6.1c), which implements 2-pass mapping to increase the chances of mapping splice reads from novel junctions $[18,19]$. To account for differences in read-lengths between batches, we created three genomic alignment index files with read lengths of 50bp, 76bp, and $101 \mathrm{bp}$, respectively, for mapping the study samples to the reference genome. The 639 binary alignment map (BAM) files containing brain RNA-seq data required additional processing before alignment and thus were converted to FASTQ files using the FastqTosam function in Picard tools [20]. Samples were checked for adaptor overabundance and overrepresented sequences using FastQC [17]. Paired-end reads were aligned to the human reference genome as described above. In order to map brain samples to the reference genome, genomic index files (read length $=101 \mathrm{bp}$ ) were created. The resulting BAM files for each brain and blood sample contained mapped paired-end reads and a corresponding alignment report file. 
Gene and isoform levels were quantified using RSEM (version 1.3.1) [21] and Bowtie2 (version 2.3.4.1) [22] and then annotated using Homo sapiens GRCh38.95.gtf annotation files. Files generated by this process for each sample contained several variables for each gene including gene id, gene length, effective gene length, expected count, counts per million (CPM), and fragments per kilobase of exon model per million reads mapped (FPKM) reads.

\section{Gene Expression Analysis in Blood and Brain}

Differential Expression Analysis: Genes with less than two reads on average among 80\% or more of the samples were excluded from analyses. Blood and brain samples were corrected for betweensample variability using a trimmed mean of M-values normalization method [23]. Differential gene expression analysis between $\mathrm{AD}$ and control subjects in blood and brain was performed separately using the VOOM and LIMMA software [24,25]. For differential gene expression analysis in brain, the normalized expression of each gene was compared between AD cases and controls using linear regression models adjusting for sex, age at death, RNA integrity number (RIN), post-mortem interval (PMI), and sequencing batch as covariates. Gene expression analysis of the blood samples included only the 179 individuals who were neuropathologically examined and models included covariates for sex, age at exam, and library batch. Analyses were performed in the total sample and subgroups defined by $A P O E$ genotype $(\varepsilon 2 / \varepsilon 3, \varepsilon 3 / \varepsilon 3$, and $\varepsilon 3 / \varepsilon 4)$. Subjects with genotypes $\varepsilon 2 / \varepsilon 2, \varepsilon 2 / \varepsilon 4$, and $\varepsilon 4 / \varepsilon 4$ were excluded from analyses due to small samples sizes (Supplementary Table 1). In blood, only samples containing a neuropathological diagnosis of $\mathrm{AD}$ were used. Analyses of gene expression in blood were further stratified by RNA batch due to differences in read-length and sample substructure (Supplementary Figure 1). For the $\varepsilon 3 / \varepsilon 4$ subgroup data from batch 2 were only analyzed because the batch 1 sample size was too small (Supplementary Table 
1). Results from analyses of each batch and $A P O E$ genotype group were combined by metaanalysis weighting for the number of $\mathrm{AD}$ cases and accounting for effect direction using the METAL program [26].

To evaluate differential gene expression patterns in the joint blood and brain datasets, we combined univariate results from blood and brain using the R package CUMP which incorporates O'Brien's method [27]. In this method, a combined z-score was calculated using t-value estimates derived from the LIMMA linear regression analyses and/or from z-score estimates from the metaanalysis of the blood batches. All analyses were weighted by the number of samples within APOE genotype groups or in the total sample.

Single Cell Gene Expression Analysis: A normalized single cell RNA-sequencing expression matrix from $\sim 2,400$ cells collected from the blood of healthy individuals and proportions of dendritic cells and monocytes for each sample was obtained from the Single Cell Portal [28]. FASTQ single nuclei RNA-sequencing data from the prefrontal cortex of 48 brains from ROSMAP participants (24 AD cases, 24 controls) were obtained from the Synapse portal [15] and processed as previously described [12]. The average expression for each cell-type in the blood and brain RNA-seq datasets was calculated for each gene.

Gene Set Enrichment Analysis: Differentially expressed genes in the total sample or within APOE genotype groups were ranked by a combined z-score from blood and brain using the O'Brien method. Gene set enrichment analysis was performed using this ranked list and gene set pathway information obtained from the Molecular Signatures Database (MSigDB) as previously described $[28,29]$. Pathway enrichment scores were determined based on the degree to which a set of genes was overrepresented by the largest positive and smallest negative z-scores. Genes that 
contributed the most to the enrichment score of each pathway were designated as leading-edge genes.

\section{Co-Expressed Gene Network Analysis in Blood and Brain}

Co-expressed genes in networks were identified using 14,456 coding genes in brain and 11,379 coding genes in blood in the ROSMAP RNA-seq dataset using the weighted gene correlation network analysis (WGCNA) algorithm [30]. Analyses of data from blood included only 141 batch 2 samples with and without post-mortem examination to avoid batch effects, and analyses of data from brain comprised 636 samples excluding lack of RIN or batch information (Supplementary

Table 2). We used gene expression levels calculated as log-transformed fragments per kilobase of transcript per million (FPKM). Soft-power parameters of 12.0 and 12.5 were selected for analyses of brain and blood data, respectively, as previously described [12]. Expression data were clustered hierarchically by implementing a dissimilatory topological overlap matrix (TOM). Initial modules with a minimal network size of 100 genes were identified and labeled using dynamic tree cutting. Eigengenes were derived from the first principle component for each module and served as representative values of gene expression in a given module [31]. Networks with high eigengene similarity and a height of 0 were merged using the mergeCloseModules function in WGCNA. Fuzzy module membership was assigned using the signedKME function.

Network modules identified in brain were examined for preservation in blood using the modulePreservation function in WGCNA. Brain networks with a Zsummary score > 5 were considered preserved in brain networks [32]. Relevance of the networks to AD pathology was established based on enrichment of $\mathrm{AD}$-related genes that was determined using the userListEnrichment function in WGCNA. For the purpose of this analysis, we defined AD-related genes which included those within $20 \mathrm{~kb}$ of single nucleotide polymorphisms (SNPs) showing at 
least modest evidence $(\mathrm{p}<0.001)$ for association with AD risk [33] or AD-related neuropathological measures of Tau and $\mathrm{A} \beta$ proteins [34]. We used EnrichR to identify KEGG pathways enriched for AD related genes in the preserved networks [35]. Next, genes in networks contributing to significant pathways were further evaluated using Ingenuity Pathway Analysis software (QIAGEN Inc.) to identify biological subnetworks.

\section{Measurements and Association with Vascular Injury Related Proteins}

Intercellular adhesion molecule 1 (ICAM-1), vascular cell adhesion molecule 1 (VCAM-1), and serum amyloid $\alpha$ (SAA) were detected and measured in fresh tissue lysate from the dorsolateral prefrontal cortex area using the Mesoscale Discovery V-PLEX Plus Vascular Injury Panel Kit (Mesoscale Discovery, K15198G, Rockville MD). Grey matter was separated from frozen brain tissue on dry ice and weighed. Ice-cold RIPA buffer (ThermoScientific, \#89901) was added to the grey matter at 5mL RIPA: $1 \mathrm{~g}$ brain wet weight, and homogenized with Qiagen Tissue Lyser LT at $50 \mathrm{~Hz}$ for five mininutes (Qiagen, Germany) (ThermoScientific, Waltham MA). The homogenate was centrifuged at $17,000 \mathrm{~g}$ at $4^{\circ} \mathrm{C}$ for 15 minutes, then the supernatant was aliquoted and stored at $-80^{\circ} \mathrm{C}$ until use. Buffers and immunoassay plates were prepared according to the manufactory instructions and the brain homogenate was further diluted 5 -fold. The immunoassay plates were read using the multi-detection SPECTOR 6000 Imager to quantitate protein levels (Mesoscale Discovery).

Additional analyses of 107 top-ranked genes emerging from pathways identified by differential gene expression and network analyses were performed using log-transformed FPKM values obtained previously from these FHS/ADRC donor brains [12]. Levels of ICAM-1, VCAM1, and SAA proteins were rank-transformed after adjusting for age and sex. We performed 
association analyses using the expression levels of the selected genes with the levels of vascular injury related proteins as quantitative outcomes in linear regression models further adjusting for RIN.

\section{Results}

\section{Differentially Expressed Genes in Blood and Brain}

Gene expression levels in 179 blood and 576 brain samples from the ROSMAP dataset were compared between AD cases and controls (Figure 1). In the total sample, no genes in the combined data from blood and brain were differentially expressed at the transcriptome-wide significance level $\left(\mathrm{P}<3.3 \times 10^{-6}\right)$. Of 78 genes associated with $\mathrm{AD}$ at a genome-wide significance level in a recent large genome-wide association study (GWAS) [36], 64 passed QC and were expressed in both brain and blood. Expression of five of these 64 genes (HLA-DQA1, INPP5D, SPDYE3, TSPOAP1, and SIGLEC11) were nominally significant $(\mathrm{P}<0.05)$ in the analysis of the combined blood and brain data (Table 1, Supplemental Table 3, Supplementary Figure 2). Differential expression of $H L A-D Q A I$ and INPP5D was nominally significant at $\mathrm{P}<0.05$ in both blood and brain with the same direction of effect. Differentially expressed genes (DEGs) after multiple testing correction at $\mathrm{P}<6.4 \times 10^{-4}$ were evident only in brain for $B C K D K\left(\mathrm{P}=5.1 \times 10^{-4}\right)$, TSPOAPI $\left(\mathrm{P}=2.6 \times 10^{-4}\right)$ and SIGLEC11 $\left(\mathrm{P}=1.6 \times 10^{-4}\right)$.

Two genes were differentially expressed between $\mathrm{AD}$ cases and controls at the transcriptome-wide level $\left(\mathrm{P}<3.3 \times 10^{-6}\right)$ within a particular APOE genotype group. PIGHP1 was significantly upregulated in $\mathrm{AD}$ cases in the combined brain and blood samples in the $\varepsilon 2 / \varepsilon 3$ group $\left(\mathrm{Z}=4.67, \mathrm{P}=3.1 \times 10^{-6}\right)$, a pattern predominated by the evidence in brain but also apparent in blood (Table 2, Supplementary Figure 2, Supplementary Figure 3a). Among $\varepsilon 3 / \varepsilon 4$ subjects, 
expression of FRAS1 was significantly downregulated in AD cases in blood only (Z=-4.66, $\mathrm{P}=3.2 \times 10^{-6}$ ) (Supplementary Figure 3b). No transcriptome-wide significant DEGs were identified in brain from any $A P O E$ genotype groups. Among genes previously associated with AD among $\varepsilon 2 / \varepsilon 3$ subjects [12], $C 4 A, C 4 B$, and $H S P A 2$ were moderately $\left(\mathrm{P}<10^{-3}\right)$ upregulated in brain but not blood from AD ROSMAP Study participants in the $\varepsilon 2 / \varepsilon 3$ subgroup and total sample (Supplemental Table 4). Notably, $C 4 B$ expression trended in the opposite direction (i.e., downregulated in $\mathrm{AD}$ cases $)$ in blood from $\varepsilon 2 / \varepsilon 3$ subjects $(\mathrm{P}=0.08)$.

Examination of cell-level expression profiles of the DEGs in Tables $\mathbf{1}$ and $\mathbf{2}$ revealed that in blood cell types $H L A-D Q A 1$ and INPP5D were more highly expressed in dendritic cells and monocytes compared to other genes in this group (Supplementary Figure 4a). INPP5D was the only gene in this group expressed in brain cell types and specifically in microglia (Supplementary Figure 4b).

\section{APOE Genotype Dependent Pathways in Combined Blood and Brain Expression Profiles}

We identified 21 pathways that were significantly enriched for upregulated or downregulated genes in the combined blood and brain expression levels in at least one $A P O E$ genotype group (Figure 2a and Supplementary Table 5). Enrichment scores from significant pathways identified in the $\varepsilon 3 / \varepsilon 4$ group were generally downregulated and had the opposite effect direction compared to those for the other $A P O E$ genotype groups (Figure 2a, Table 3). Six pathways were significantly and uniquely enriched in the $\varepsilon 3 / \varepsilon 4$ subgroup including apoptosis, estrogen response late, hypoxia, il6/jak/stat3 signaling, inflammatory response, and p53 pathway. Pathways for allograft rejection, interferon gamma response, peroxisome and TNFA signaling via NFKB were enriched for upregulated $\mathrm{AD}$ genes in the $\varepsilon 3 / \varepsilon 4$ group but for downregulated $\mathrm{AD}$ genes in the $\varepsilon 2 / \varepsilon 3$ 
and $\varepsilon 3 / \varepsilon 3$ groups. There was little overlap of the leading-edge genes for these four pathways across $A P O E$ genotype groups with at most one overlapping gene per pathway (Table 3). Leading-edge genes in the same pathway shared by different APOE genotypes included HLA-DRA in allograft rejection, $C D 74$ in interferon gamma response, and $K Y N U$ in TNFA signaling via NFKB. Among 92 leading-edge genes from the four pathways that had enrichment scores in opposite directions among subjects with and without $\varepsilon 4$ (Table 3), expression of 21 genes in FHS/ADRC brains was significantly associated $\left(\mathrm{P}<4.67 \times 10^{-4}\right)$ with at least one of the three vascular injury related proteins (Figure 2b, Table 4). Expression of 11 of these 21 genes (52\%) was significantly associated with SAA level, and expression of 13 genes (62\%) was significantly associated with VCAM-1 level. Expression of only two genes, TRIP10, and FOSL1, which are both involved in signaling via NFKB, was significantly associated with ICAM-1 levels.

\section{Co-Expression Networks Common to Brain and Blood}

Four co-expression networks identified in brain were preserved in blood (Supplementary Table 6). The eigengene value (i.e., first principle component of gene expression across the network) in the greenyellow network was significantly higher among $\varepsilon 4$ carriers than non-carriers $\left(\mathrm{P}=4.7 \times 10^{-}\right.$

$\left.{ }^{3}\right)$ (Figure 3a). The greenyellow network is significantly enriched for genes previously associated with AD risk [33] and plaque score [34] (Supplementary Table 6). The AD-related genes in this network were significantly enriched in nine KEGG pathways (Figure 3b, Supplementary Table 7). Seventeen genes contributing to these significant pathways form a biological subnetwork (Figure 3c). One of these genes, NFKBIA, is a leading-edge gene from the signaling via NFKB pathway and was involved in five out of nine significant pathways in the greenyellow network (Table 3, Supplementary Table 7). HLA-DRA is involved in six of the nine significant pathways 
in the greenyellow network and is a leading-edge gene in the allograft rejection pathway identified in the $\varepsilon 3 / \varepsilon 3$ and $\varepsilon 3 / \varepsilon 4$ groups. INPP5D, which is differentially expressed in both blood and brain (Table 1, Supplementary Figure 2), is involved in two significant KEGG pathways (Fc gamma $R$-mediated phagocytosis and $B$ cell receptor signaling) in the greenyellow network. $C 4 B$, which is upregulated in brain from $\mathrm{AD}$ cases compared to controls in the $\varepsilon 2 / \varepsilon 3$ group (Supplementary

Table 4), was included in the greenyellow network pathways involved in staphylococcus aureus infection and systemic lupus erythematosus.

Two of the 17 sub-network genes in the significant pathways enriched for AD genes in the greenyellow network (Supplementary Table 8) were significantly associated with the level of at least one of the three vascular injury proteins after multiple testing correction (Figure 3d). Specifically, VASP expression was significantly associated with levels of ICAM-1 ( $\left.\mathrm{P}=3.7 \times 10^{-4}\right)$ and SAA $\left(\mathrm{P}=1.0 \times 10^{-4}\right)$, and $C 4 B$ expression was significantly associated with levels of ICAM-1 $\left(\mathrm{P}=1.3 \times 10^{-5}\right)$, SAA $\left(\mathrm{P}=1.6 \times 10^{-4}\right)$ and VCAM-1 $\left(\mathrm{P}=7.0 \times 10^{-6}\right)$.

\section{Discussion}

The primary purpose of this study was to identify genes previously associated with $\mathrm{AD}$ and in biological pathways enriched for $\mathrm{AD}$ genes whose expression differs between $\mathrm{AD}$ cases and controls in both blood and brain, especially in an $A P O E$ genotype-specific manner. We observed that two established $\mathrm{AD}$ genes, INPP5D and HLA-DQA1, were differentially expressed in both blood and brain. Among the 21 top-ranked pathways in the combined blood and brain expression profiles, 10 pathways were specific to persons having the APOE $\varepsilon 3 / \varepsilon 4$ genotype. Additionally, we identified a co-expression network enriched for AD genes in brain that was preserved in blood and showed significantly higher average expression in $\varepsilon 4$ carriers than non-carriers. Lastly, several genes from the top-ranked pathways and co-expression networks were significantly associated 
with levels of vascular injury proteins. These findings suggest that $\mathrm{AD}$ genes that are differentially expressed in both blood and brain and associated with vascular markers may be involved in BBB function, and their effects are dependent on $A P O E$ genotypes.

The BBB is a semi-permeable endothelial cell membrane regulating transport between cerebral blood vessels and the central nervous system [37]. The dysregulation of the BBB has been implicated in early cognitive decline and exacerbation of neuroinflammation and neurodegeneration [38]. A recent study showed that APOE $\varepsilon 4$ carriers exhibit BBB dysfunction and cognitive decline independent of AD pathology [8]. Our analyses identified six pathways uniquely enriched for DEGs among $\varepsilon 3 / \varepsilon 4$ carriers in combined blood and brain expression data. Expression of INPP5D and HLA-DQA-1 was significantly greater in both blood and brain from individuals with $\mathrm{AD}$ compared to controls. Increased expression of INPP5D in blood has been previously linked with increased risk of hemorrhagic transformation [39], which is associated also with BBB permeability [40]. INPP5D is highly expressed in microglia and encodes the protein SHIP1 which has been implicated in many neuroinflammatory processes [41]. Additionally, HLA$D Q A I$ and INPP5D are expressed in dendritic cells and monocytes and involved in immune processes, and the migration of monocytes across an inflamed BBB can cause differentiation into dendritic cells [42]. FRAS1 was significantly downregulated in AD compared to controls in blood from $\varepsilon 3 / \varepsilon 4 \mathrm{AD}$ individuals and a recent study showed that FRAS1 knockdown mice were impaired in memory and learning behaviors [43].

We identified four pathways (allograft rejection, interferon gamma response, peroxisome, and TNFA signaling via NFKB) containing gene sets that, with respect to $\mathrm{AD}$, were significantly upregulated in blood and brain from $\varepsilon 4$ carriers and other gene sets from the same pathways that were downregulated in individuals without $\varepsilon 4$. The inflammatory cytokine interferon gamma has 
been shown to impact directly brain endothelium to cause BBB breakdown [44] and can inhibit ApoE production in macrophages [45]. Peroxisomes synthesize fatty acids which have been implicated in the development of AD [46]. The TNFA via NFKB signaling pathway has been implicated in BBB dysfunction [47], and the TNFA and NFKB pathways have been independently associated with increased neuroinflammation related to APOE $\varepsilon 4[48,49]$.

Multiple genes from networks we observed to be preserved in brain and blood transcriptome data and enriched in pathways from combined blood and brain expression profiles showed significant association with the vascular injury proteins ICAM-1, SAA, and VCAM-1. SAA level increases in the presence of BBB dysfunction [50]. ICAM-1 is a cytokine involved regulation of the BBB [51] and increased ICAM-1 level has been associated with BBB damage and neuroinflammation [52]. Under inflammatory conditions, VCAM-1 level is upregulated and the BBB can release soluble VCAM-1 which in turn can compromise BBB function [53]. Our study showed that FOSL1 and TRIP10 were among the genes enriched in the TNFA via NFKB pathway, and their expression was associated with ICAM-1. TRIP10 was previously included in an AD network derived from multi-omic integration [54] and FOSL-1 was identified in conjunction with PIAS1, a protein associated with AD and inflammatory response [55]. We identified $V A S P$ and $C 4 B$ in an $A P O E$ genotype-specific co-expressed gene network in brain that was reproduced in blood, and expression of these genes was significantly associated with levels of multiple vascular damage proteins. VASP encodes vasodilator-stimulated phosphoprotein which regulates $\mathrm{BBB}$ function [56]. The pattern of $C 4 B$ expression in brain is dependent on $A P O E$ genotype [12] and dysregulation of complement system can cause or exacerbate BBB dysfunction [57]. These genes require further investigation in their role with $\mathrm{AD}$ specifically related to $\mathrm{BBB}$ function and $A P O E$. 


\section{Limitations}

Our study has several limitations. First, the sample sizes of the APOE genotype groups in the blood dataset were relatively small which limited statistical power. Additionally, the ROSMAP blood dataset exhibited significant batch effects. However, we were able to account for these batch effects by running each batch separately and meta-analyzing our results. Second, the software WGCNA creates networks based on strong computational correlations but does not account for underlying biological implications. We evaluated biological connections using the IPA software by rebuilding subnetworks of the leading-edge genes. Third, we were able to obtain single-cell data only for dendritic cells and monocytes in blood and therefore could not analyze a wider array of blood cell types.

\section{Conclusions}

Our study provides evidence of the importance of evaluating brain and blood transcriptome data together with genetic information derived from the same subjects to identify meaningful correlations of blood biomarkers with AD-related proteins in brain. Future studies are required to investigate further, how the genes and biological pathways identified in this study in the context of $A P O E$ genotype influence the $\mathrm{BBB}$ and contribute to and/or exacerbate AD-related pathology.

\section{List of abbreviations:}

A $\beta$ : amyloid- $\beta$, AD: Alzheimer's Disease, BBB: Blood-Brain Barrier, CAA: cerebral amyloid angiopathy, CERAD: Consortium to Establish a Registry for Alzheimer Disease, CPM: counts per million, DE: differential expression, DGE: Differential gene expression, FHS/ADRC: Framingham Heart Study/Boston University Alzheimer's Disease Research Center, FPKM: 
fragments per kilobase of transcript per million, GWAS: genome-wide association study, ICAM1: Intercellular adhesion molecule 1, PMI: post mortem interval, RIN: RNA integrity number, ROSMAP - Religious Orders Study and Rush Memory and Aging Project, RNA-seq: RNA sequencing, SAA : serum amyloid $\alpha$, VCAM-1: vascular cell adhesion molecule 1, WGCNA: weighted gene correlation network analysis

\section{Acknowledgements}

Not applicable

\section{Funding}

This study was supported by the National Institute on Aging (NIA) grants, RF1-AG057519, R01AG069453, U01-AG068057, U19-AG068753, R01-AG048927， U01-AG062602， U01AG058654, RF1-AG057768, RF1-AG054156, P30-AG072978, U01-AG032984, and RF1AG063913. Collection of study data provided by the Rush Alzheimer's Disease Center, Rush University Medical Center, Chicago was supported through funding by NIA grants P30-AG10161, R01-AG15819, R01-AG17917, R01-AG30146, R01-AG36836, U01-AG32984, U01-AG46152, and U01-AG61358, and funding from the Illinois Department of Public Health and the Translational Genomics Research Institute.

\section{Availability of data and materials}

The datasets supporting the conclusions of this article are available in https://singlecell.broadinstitute.org/ and http://www.synapse.org, as well as from the corresponding author upon request. 


\section{Authors' contributions}

R.P., L.A.F., and G.R.J. conceived overall study design. R.P. and G.R.J. perceived statistical analysis. R.P. and J.H. performed data analyses. W.X. and T.D.S. conducted immunoassay experiments using autopsied brains. D.A.B. and T.D.S. provided neuropathological data. R.P., D.A.B., T.D.S., W.X., L.A.F., and G.R.J. reviewed and edited the manuscript. G.R.J. and L.A.F. supervised and obtained funding for the project.

\section{Ethics approval and consent to participate}

The study protocol, design, and performance of the current study were approved by the Boston University Institutional Review Board.

\section{Consent for publication}

Not applicable

\section{Competing interests}

The authors declare no competing interests.

\section{Figure Legends}

Figure 1. Analysis design and workflow. RNA-sequencing data were obtained from blood and post-mortem frozen brain of neuropathologically verified AD cases and controls. Data were analyzed in two ways. First, gene co-expression analysis identified networks in brain that reproduced in blood. A second analysis identified genes differentially expressed between AD cases 
in controls in the total sample as well as within $A P O E$ genotype groups in both blood and brain. Expression of genes in the co-expression networks that were previously associated with AD by GWAS were tested for associated with AD-related traits measured in brain. Next, genes in significant co-expression networks and differentially expressed genes in blood and brain were incorporated as seeds in pathway analysis. Finally, expression of genes from the most significant pathways was tested for association with levels of several vascular damage proteins. Figure created with BioRender.com

Figure 2. Significant pathways in blood and brain by $A P O E$ genotype. (a) Significant pathways (adjusted $\mathrm{P}<0.05$ ) within $A P O E$ genotype groups that are enriched for differentially expressed genes in blood and brain combined are plotted according to the normalized enrichment score. Enrichment score indicates whether the genes in the pathway are upregulated (positive) or downregulated (negative) in AD. (b) Heatmap shows strength and direction of association of levels of proteins involved in vascular damage with expression of leading-edge genes from significant pathways where enrichment scores are in opposite directions between APOE \&4 carriers (+) and non-carriers (-). Genes whose expression was significantly $\left(\mathrm{P}<4.67 \times 10^{-4}\right)$ associated with the level of at least one protein (indicated by an asterisk) are shown.

Figure 3. APOE genotype-specific co-expression networks in blood and brain. (a) Boxplot for the greenyellow network showing the distribution of eigengene values, which summarize gene expression across a network, among APOE $\varepsilon 4$ carriers (+) and non-carriers (-). P-value was calculated using the student's t-test. (b) Barplot showing significant pathways enriched for established AD genes in the greenyellow network. (c) Biological subnetwork including established 
AD genes involved in significant pathways in the greenyellow network. (d) Heatmap showing strength of association of seed-gene expression in brain from the biological subnetwork in (c) with levels of proteins involved in vascular damage. Asterisks indicate significant associations $\left(\mathrm{P}<4.67 \times 10^{-4}\right)$.

\section{References}

1. DeTure MA, Dickson DW. The neuropathological diagnosis of Alzheimer's disease. Molecular Neurodegeneration. 2019;14:32.

2. Fehlbaum-Beurdeley P, Jarrige-Le Prado AC, Pallares D, Carrière J, Guihal C, Soucaille C, et al. Toward an Alzheimer's disease diagnosis via high-resolution blood gene expression. Alzheimer's \& Dementia. 2010;6:25-38.

3. Zetterberg H, Burnham SC. Blood-based molecular biomarkers for Alzheimer's disease. Molecular Brain. 2019;12:26.

4. Bell RD, Zlokovic BV. Neurovascular mechanisms and blood-brain barrier disorder in Alzheimer's disease. Acta Neuropathol. 2009;118:103-13.

5. Cai Z, Qiao P-F, Wan C-Q, Cai M, Zhou N-K, Li Q. Role of Blood-Brain Barrier in Alzheimer's Disease. Journal of Alzheimer's Disease. IOS Press; 2018;63:1223-34.

6. Ramos-Cejudo J, Wisniewski T, Marmar C, Zetterberg H, Blennow K, de Leon MJ, et al. Traumatic Brain Injury and Alzheimer's Disease: The Cerebrovascular Link. EBioMedicine. 2018;28:21-30.

7. Farrer LA, Cupples LA, Haines JL, Hyman B, Kukull WA, Mayeux R, et al. Effects of age, sex, and ethnicity on the association between apolipoprotein E genotype and Alzheimer disease. A 
meta-analysis. APOE and Alzheimer Disease Meta Analysis Consortium. JAMA. 1997;278:1349-56.

8. Montagne A, Nation DA, Sagare AP, Barisano G, Sweeney MD, Chakhoyan A, et al. APOE4 leads to blood-brain barrier dysfunction predicting cognitive decline. Nature. Nature Publishing Group; 2020;581:71-6.

9. Corder EH, Saunders AM, Strittmatter WJ, Schmechel DE, Gaskell PC, Small GW, et al. Gene dose of apolipoprotein E type 4 allele and the risk of Alzheimer's disease in late onset families. Science. 1993;261:921-3.

10. Reiman EM, Arboleda-Velasquez JF, Quiroz YT, Huentelman MJ, Beach TG, Caselli RJ, et al. Exceptionally low likelihood of Alzheimer's dementia in APOE2 homozygotes from a 5,000-person neuropathological study. Nat Commun. 2020;11:1-11.

11. Yu L, Boyle PA, Nag S, Leurgans S, Buchman AS, Wilson RS, et al. APOE and cerebral amyloid angiopathy in community-dwelling older persons. Neurobiol Aging. 2015;36:294653.

12. Panitch R, Hu J, Chung J, Zhu C, Meng G, Xia W, et al. Integrative Brain Transcriptome Analysis Links Complement Component 4 and HSPA2 to the APOE $\varepsilon 2$ Protective Effect in Alzheimer Disease. medRxiv. Cold Spring Harbor Laboratory Press; 2020;2020.11.23.20235762.

13. Jun G, You Y, Zhu C, Meng G, Chung J, Panitch R, et al. Protein phosphatase 2A, complement component 4, and APOE genotype linked to Alzheimer disease using a systems biology approach. medRxiv. Cold Spring Harbor Laboratory Press; 2020;2020.11.20.20235051.

14. Bennett DA, Buchman AS, Boyle PA, Barnes LL, Wilson RS, Schneider JA. Religious Orders Study and Rush Memory and Aging Project. J Alzheimers Dis. 2018;64:S161-89. 
15. De Jager PL, Ma Y, McCabe C, Xu J, Vardarajan BN, Felsky D, et al. A multi-omic atlas of the human frontal cortex for aging and Alzheimer's disease research. Sci Data. 2018;5:180142.

16. Hyman BT, Phelps CH, Beach TG, Bigio EH, Cairns NJ, Carrillo MC, et al. National Institute on Aging-Alzheimer's Association guidelines for the neuropathologic assessment of Alzheimer's disease. Alzheimers Dement. 2012;8:1-13.

17. Babraham Bioinformatics - FastQC A Quality Control tool for High Throughput Sequence Data [Internet]. [cited 2019 Sep 6]. Available from: http://www.bioinformatics.babraham.ac.uk/projects/fastqc/

18. Dobin A, Davis CA, Schlesinger F, Drenkow J, Zaleski C, Jha S, et al. STAR: ultrafast universal RNA-seq aligner. Bioinformatics. Oxford University Press; 2013;29:15.

19. Dobin A, Gingeras TR. Mapping RNA-seq Reads with STAR. Curr Protoc Bioinformatics. 2015;51:11.14.1-11.14.19.

20. Picard Tools - By Broad Institute [Internet]. [cited 2020 Dec 17]. Available from: http://broadinstitute.github.io/picard/

21. Li B, Dewey CN. RSEM: accurate transcript quantification from RNA-Seq data with or without a reference genome. BMC Bioinformatics. 2011;12:323.

22. Langmead B, Salzberg SL. Fast gapped-read alignment with Bowtie 2. Nat Methods. 2012;9:357-9.

23. Robinson MD, Oshlack A. A scaling normalization method for differential expression analysis of RNA-seq data. Genome Biology. 2010;11:R25.

24. Law CW, Chen Y, Shi W, Smyth GK. voom: precision weights unlock linear model analysis tools for RNA-seq read counts. Genome Biology. 2014;15:R29. 
25. Ritchie ME, Phipson B, Wu D, Hu Y, Law CW, Shi W, et al. limma powers differential expression analyses for RNA-sequencing and microarray studies. Nucleic Acids Res. 2015;43:e47-e47.

26. Willer CJ, Li Y, Abecasis GR. METAL: fast and efficient meta-analysis of genomewide association scans. Bioinformatics. 2010;26:2190-1.

27. Yang Q, Wang Y. Methods for Analyzing Multivariate Phenotypes in Genetic Association Studies. Shao Y, editor. Journal of Probability and Statistics. Hindawi Publishing Corporation; 2012;2012:652569.

28. Korotkevich G, Sukhov V, Sergushichev A. Fast gene set enrichment analysis. bioRxiv. Cold Spring Harbor Laboratory; 2019;060012.

29. Subramanian A, Tamayo P, Mootha VK, Mukherjee S, Ebert BL, Gillette MA, et al. Gene set enrichment analysis: A knowledge-based approach for interpreting genome-wide expression profiles. PNAS. National Academy of Sciences; 2005;102:15545-50.

30. Langfelder P, Horvath S. WGCNA: an R package for weighted correlation network analysis. BMC Bioinformatics. 2008;9:559.

31. Langfelder P, Horvath S. Eigengene networks for studying the relationships between coexpression modules. BMC Systems Biology. 2007;1:54.

32. Bakhtiarizadeh MR, Hosseinpour B, Shahhoseini M, Korte A, Gifani P. Weighted Gene Coexpression Network Analysis of Endometriosis and Identification of Functional Modules Associated With Its Main Hallmarks. Front Genet. 2018;9:453.

33. Kunkle BW, Grenier-Boley B, Sims R, Bis JC, Damotte V, Naj AC, et al. Genetic metaanalysis of diagnosed Alzheimer's disease identifies new risk loci and implicates $A \beta$, tau, immunity and lipid processing. Nat Genet. 2019;51:414-30. 
34. Beecham GW, Hamilton K, Naj AC, Martin ER, Huentelman M, Myers AJ, et al. GenomeWide Association Meta-analysis of Neuropathologic Features of Alzheimer's Disease and Related Dementias. PLOS Genetics. Public Library of Science; 2014;10:e1004606.

35. Chen EY, Tan CM, Kou Y, Duan Q, Wang Z, Meirelles GV, et al. Enrichr: interactive and collaborative HTML5 gene list enrichment analysis tool. BMC Bioinformatics. 2013;14:128.

36. Bellenguez C, Küçükali F, Jansen I, Andrade V, Moreno-Grau S, Amin N, et al. New insights on the genetic etiology of Alzheimer's and related dementia. medRxiv. Cold Spring Harbor Laboratory Press; 2020;2020.10.01.20200659.

37. Abbott NJ, Patabendige AAK, Dolman DEM, Yusof SR, Begley DJ. Structure and function of the blood-brain barrier. Neurobiol Dis. 2010;37:13-25.

38. Zenaro E, Piacentino G, Constantin G. The blood-brain barrier in Alzheimer's disease. Neurobiol Dis. 2017;107:41-56.

39. Jickling GC, Ander BP, Stamova B, Zhan X, Liu D, Rothstein L, et al. RNA in blood is altered prior to hemorrhagic transformation in ischemic stroke. Ann Neurol. 2013;74:232-40.

40. Rosenthal SL, Kamboh MI. Late-Onset Alzheimer's Disease Genes and the Potentially Implicated Pathways. Curr Genet Med Rep. 2014;2:85-101.

41. Malik M, Parikh I, Vasquez JB, Smith C, Tai L, Bu G, et al. Genetics ignite focus on microglial inflammation in Alzheimer's disease. Mol Neurodegener [Internet]. 2015 [cited 2021 May 3];10. Available from: https://www.ncbi.nlm.nih.gov/pmc/articles/PMC4595327/

42. Ifergan I, Kébir H, Bernard M, Wosik K, Dodelet-Devillers A, Cayrol R, et al. The bloodbrain barrier induces differentiation of migrating monocytes into Th17-polarizing dendritic cells. Brain. 2008;131:785-99. 
43. Kalpachidou T, Makrygiannis AK, Pavlakis E, Stylianopoulou F, Chalepakis G, Stamatakis A. Behavioural effects of extracellular matrix protein Fras1 depletion in the mouse. Eur J Neurosci. 2020;

44. Bonney S, Seitz S, Ryan CA, Jones KL, Clarke P, Tyler KL, et al. Gamma Interferon Alters Junctional Integrity via Rho Kinase, Resulting in Blood-Brain Barrier Leakage in Experimental Viral Encephalitis. mBio. 2019;10.

45. Brand K, Mackman N, Curtiss LK. Interferon-gamma inhibits macrophage apolipoprotein E production by posttranslational mechanisms. J Clin Invest. 1993;91:2031-9.

46. Lizard G, Rouaud O, Demarquoy J, Cherkaoui-Malki M, Iuliano L. Potential roles of peroxisomes in Alzheimer's disease and in dementia of the Alzheimer's type. J Alzheimers Dis. 2012;29:241-54.

47. Coelho-Santos V, Leitão RA, Cardoso FL, Palmela I, Rito M, Barbosa M, et al. The TNF$\alpha / \mathrm{NF}-\kappa \mathrm{B}$ signaling pathway has a key role in methamphetamine-induced blood-brain barrier dysfunction. J Cereb Blood Flow Metab. 2015;35:1260-71.

48. Ophir G, Amariglio N, Jacob-Hirsch J, Elkon R, Rechavi G, Michaelson DM. Apolipoprotein E4 enhances brain inflammation by modulation of the NF-kappaB signaling cascade. Neurobiol Dis. 2005;20:709-18.

49. Fan Y-Y, Cai Q-L, Gao Z-Y, Lin X, Huang Q, Tang W, et al. APOE $\varepsilon 4$ allele elevates the expressions of inflammatory factors and promotes Alzheimer's disease progression: A comparative study based on Han and She populations in the Wenzhou area. Brain Res Bull. 2017;132:39-43. 
50. Bowman GL, Dayon L, Kirkland R, Wojcik J, Peyratout G, Severin IC, et al. Blood-brain barrier breakdown, neuroinflammation, and cognitive decline in older adults. Alzheimer's \& Dementia. 2018;14:1640-50.

51. Dietrich J-B. The adhesion molecule ICAM-1 and its regulation in relation with the bloodbrain barrier. J Neuroimmunol. 2002;128:58-68.

52. Huber JD, Campos CR, Mark KS, Davis TP. Alterations in blood-brain barrier ICAM-1 expression and brain microglial activation after $\lambda$-carrageenan-induced inflammatory pain. Am J Physiol Heart Circ Physiol. 2006;290:H732-40.

53. Haarmann A, Nowak E, Deiß A, van der Pol S, Monoranu C-M, Kooij G, et al. Soluble VCAM1 impairs human brain endothelial barrier integrity via integrin $\alpha$-4-transduced outside-in signalling. Acta Neuropathol. 2015;129:639-52.

54. Klein H-U, Schäfer M, Bennett DA, Schwender H, De Jager PL. Bayesian integrative analysis of epigenomic and transcriptomic data identifies Alzheimer's disease candidate genes and networks. PLoS Comput Biol [Internet]. 2020 [cited 2021 Apr 9];16. Available from: https://www.ncbi.nlm.nih.gov/pmc/articles/PMC7138305/

55. He K, Zhang J, Liu J, Cui Y, Liu LG, Ye S, et al. Functional genomics study of protein inhibitor of activated STAT1 in mouse hippocampal neuronal cells revealed by RNA sequencing. Aging (Albany NY). 2021;13:9011-27.

56. Kraft P, Benz PM, Austinat M, Brede ME, Schuh K, Walter U, et al. Deficiency of VasodilatorStimulated Phosphoprotein (VASP) Increases Blood-Brain-Barrier Damage and Edema Formation after Ischemic Stroke in Mice. PLOS ONE. Public Library of Science; 2010;5:e15106. 
57. Alexander JJ. Blood-brain barrier (BBB) and the complement landscape. Molecular Immunology. 2018;102:26-31. 
Table 1. Differentially expressed known AD genes in the combined blood and brain datasets in the total sample.

\begin{tabular}{|c|c|c|c|c|c|c|c|c|c|c|c|c|c|}
\hline \multirow{2}{*}{ Gene } & \multirow{2}{*}{ Dataset } & \multicolumn{3}{|c|}{ APOE $\varepsilon 2 / \varepsilon 3$} & \multicolumn{3}{|c|}{ APOE $\varepsilon 3 / \varepsilon 3$} & \multicolumn{3}{|c|}{ APOE $\varepsilon 3 / \varepsilon 4$} & \multicolumn{3}{|c|}{ Total } \\
\hline & & $\mathbf{N}$ & Z-score & P-value & $\mathbf{N}$ & Z-score & P-value & $\mathbf{N}$ & Z-score & P-value & $\mathbf{N}$ & Z-score & P-value \\
\hline \multirow{3}{*}{ INPP5D } & Blood & 26 & -0.62 & 0.53 & 102 & 1.67 & 0.10 & 31 & 2.12 & 0.03 & 179 & 2.16 & 0.03 \\
\hline & Brain & 71 & 1.80 & 0.07 & 355 & 0.92 & 0.36 & 126 & 0.66 & 0.51 & 576 & 2.30 & 0.02 \\
\hline & Combined & 97 & 1.51 & 0.13 & 457 & 1.29 & 0.20 & 157 & 1.31 & 0.19 & 755 & 2.42 & 0.02 \\
\hline \multirow{3}{*}{ HLA-DQA1 } & Blood & 26 & -1.49 & 0.14 & 102 & -1.80 & 0.07 & 31 & 0.96 & 0.34 & 179 & -2.26 & 0.03 \\
\hline & Brain & 71 & 0.30 & 0.76 & 355 & -3.13 & $1.7 \times 10^{-3}$ & 126 & -1.01 & 0.31 & 576 & -2.23 & 0.03 \\
\hline & Combined & 97 & -0.16 & 0.87 & 457 & -2.84 & $4.6 \times 10^{-3}$ & 157 & -0.66 & 0.51 & 755 & -2.42 & 0.02 \\
\hline \multirow{3}{*}{ SPDYE3 } & Blood & 26 & -0.08 & 0.94 & 102 & -0.76 & 0.45 & 31 & -0.96 & 0.34 & 179 & -1.47 & 0.14 \\
\hline & Brain & 71 & -0.79 & 0.43 & 355 & -0.97 & 0.33 & 126 & -2.16 & 0.03 & 576 & -2.73 & $6.4 \times 10^{-3}$ \\
\hline & Combined & 97 & -0.77 & 0.44 & 457 & -0.96 & 0.33 & 157 & -2.37 & 0.02 & 755 & -2.26 & 0.02 \\
\hline \multirow{3}{*}{ TSPOAP1 } & Blood & 26 & -1.38 & 0.17 & 102 & 0.30 & 0.77 & 31 & -0.16 & 0.87 & 179 & -0.55 & 0.58 \\
\hline & Brain & 71 & -1.04 & 0.30 & 355 & -2.17 & 0.03 & 126 & -1.85 & 0.06 & 576 & -3.65 & $2.6 \times 10^{-4}$ \\
\hline & Combined & 97 & -1.39 & 0.16 & 457 & -1.35 & 0.18 & 157 & -1.82 & 0.07 & 755 & -2.24 & 0.03 \\
\hline \multirow{3}{*}{ SIGLEC11 } & Blood & 26 & 1.43 & 0.15 & 102 & 0.47 & 0.64 & 31 & 0.24 & 0.81 & 179 & 0.33 & 0.74 \\
\hline & Brain & 71 & 1.05 & 0.29 & 355 & 2.46 & 0.01 & 126 & 2.58 & $9.9 \times 10^{-3}$ & 576 & 3.77 & $1.6 \times 10^{-4}$ \\
\hline & Combined & 97 & 1.42 & 0.16 & 457 & 1.86 & 0.06 & 157 & 2.54 & 0.01 & 755 & 2.17 & 0.03 \\
\hline
\end{tabular}


Table 2. Novel differentially expressed genes in blood or brain within APOE genotype groups

\begin{tabular}{|c|c|c|c|c|c|c|c|c|c|c|c|c|c|}
\hline \multirow[b]{2}{*}{ Gene } & \multirow[b]{2}{*}{ Dataset } & \multicolumn{3}{|c|}{ APOE $\varepsilon 2 / \varepsilon 3$} & \multicolumn{3}{|c|}{ APOE $\varepsilon 3 / \varepsilon 3$} & \multicolumn{3}{|c|}{ APOE $\varepsilon 3 / \varepsilon 4$} & \multicolumn{3}{|l|}{ Total } \\
\hline & & $\mathbf{N}$ & $\begin{array}{l}\text { Z- } \\
\text { score }\end{array}$ & P-value & $\mathbf{N}$ & $\begin{array}{l}\text { Z- } \\
\text { score }\end{array}$ & P-value & $\mathbf{N}$ & $\begin{array}{l}\text { Z- } \\
\text { score }\end{array}$ & P-value & $\mathbf{N}$ & $\begin{array}{l}\text { Z- } \\
\text { score }\end{array}$ & P-value \\
\hline \multirow{3}{*}{ FRAS1 } & Blood & NA & NA & NA & 75 & -0.24 & 0.81 & 31 & -4.66 & $3.2 \times 10^{-6}$ & 132 & -2.26 & 0.02 \\
\hline & Brain & NA & NA & NA & 355 & -0.97 & 0.33 & 126 & 0.28 & 0.78 & 576 & -0.49 & 0.62 \\
\hline & Combined & NA & NA & NA & 430 & -0.75 & 0.45 & 157 & -1.23 & 0.22 & 708 & -1.27 & 0.20 \\
\hline \multirow{3}{*}{ PIGHP1 } & Blood & 26 & 1.67 & 0.09 & 102 & 0.43 & 0.67 & 31 & 1.30 & 0.19 & 179 & 1.14 & 0.25 \\
\hline & Brain & 71 & 4.42 & $9.8 \times 10^{-6}$ & 355 & 0.53 & 0.59 & 126 & 0.47 & 0.64 & 576 & 1.98 & 0.05 \\
\hline & Combined & 97 & 4.67 & $3.1 \times 10^{-6}$ & 457 & 0.53 & 0.59 & 157 & 0.87 & 0.39 & 755 & 1.68 & 0.09 \\
\hline
\end{tabular}

NA: not available due to low expression. Bolded p-values pass transcriptome-wide multiple testing threshold $\left(3.3 \times 10^{-6}\right)$. 
Table 3. Significant co-expressed gene pathways in the combined blood and brain datasets

\begin{tabular}{|c|c|c|c|c|}
\hline $\begin{array}{l}\text { Hallmark } \\
\text { Pathway }\end{array}$ & $\begin{array}{l}\text { APOE } \\
\text { genotype }\end{array}$ & NES & $\begin{array}{l}\text { Adjusted } \\
\text { P-value }\end{array}$ & Leading Edge Genes \\
\hline \multirow{2}{*}{$\begin{array}{l}\text { Allograft } \\
\text { rejection }\end{array}$} & $\varepsilon 3 / \varepsilon 3$ & -1.89 & 0.02 & $\begin{array}{l}\text { C2, HLA-DQA1, FAS, HLA-A, UBE2N, HLA-DOB, LTB, F2R, HLA- } \\
\text { DRA, TAP2, B2M, CD1D, CD74, MAP3K7 }\end{array}$ \\
\hline & $\varepsilon 3 / \varepsilon 4$ & 2.16 & $7.4 \times 10^{-3}$ & $\begin{array}{l}\text { IRF4, CCL22, IRF7, CD74, HLA-DRA, ELF4, IL16, IFNGR2, IL27RA, } \\
\text { IL1B }\end{array}$ \\
\hline \multirow{2}{*}{$\begin{array}{l}\text { Interferon } \\
\text { gamma response }\end{array}$} & $\varepsilon 3 / \varepsilon 3$ & -2.56 & $1.3 \times 10^{-5}$ & $\begin{array}{l}\text { HLA-DQA1, FAS, CFB, BPGM, C1S, HLA-A, LAP3, MVP, PSME2, } \\
\text { PSMA2, UBE2L6, SERPING1, DHX58, IFITM2, CD38, B2M, CD74 }\end{array}$ \\
\hline & $\varepsilon 3 / \varepsilon 4$ & 1.80 & 0.04 & $\begin{array}{l}\text { CD274, CD69, BTG1, ISG20, PML, IRF4, NFKBIA, IRF7, CD74, IL10RA, } \\
\text { IRF9 }\end{array}$ \\
\hline \multirow{2}{*}{ Peroxisome } & $\varepsilon 3 / \varepsilon 3$ & -1.81 & 0.03 & IDH2, EHHADH, MVP, ALDH1A1, SCP2, SOD1, ABCD2, MSH2 \\
\hline & $\varepsilon 3 / \varepsilon 4$ & 1.90 & 0.03 & RDH11, ELOVL5, SLC25A19, CTPS1, SLC23A2, SEMA3C \\
\hline \multirow[b]{2}{*}{$\begin{array}{l}\text { Tnfa signaling } \\
\text { via nfkb }\end{array}$} & $\varepsilon 2 / \varepsilon 3$ & -2.03 & 0.01 & $\begin{array}{l}\text { DUSP4, NR4A1, NR4A3, MARCKS, NFAT5, PHLDA1, DUSP2, KYNU, } \\
\text { G0S2, ETS2, PTGS2, GCH1, MSC, SOD2, EGR2 }\end{array}$ \\
\hline & $\varepsilon 3 / \varepsilon 4$ & 2.27 & $2.5 \times 10^{-3}$ & $\begin{array}{l}\text { TRIP10, CD69, BTG1, DENND5A, PFKFB3, FOS, NFKBIA, LDLR, } \\
\text { IER2, JUN, IL1A, PANX1, PNRC1, DUSP1, IFNGR2, OLR1, MAFF, } \\
\text { IL1B, TNIP2, CCL20, BIRC2, IER3, GADD45B, KYNU, LITAF, CCRL2, } \\
\text { SPHK1, FOSL1 }\end{array}$ \\
\hline
\end{tabular}

NES = Normalized Enrichment Score 
Table 4. Association of expression of leading-edge genes from co-expressed gene networks with vascular damage protein levels

\begin{tabular}{|c|c|c|c|c|c|c|c|c|}
\hline \multirow{2}{*}{ Gene } & \multirow{2}{*}{$\begin{array}{l}\text { APOE } \\
\text { Genotype }\end{array}$} & \multirow{2}{*}{ Pathway(s) } & \multicolumn{2}{|c|}{ I-CAM1 } & \multicolumn{2}{|c|}{ SAA } & \multicolumn{2}{|c|}{ V-CAM1 } \\
\hline & & & $\beta$ & P-value & $\beta$ & P-value & $\beta$ & P-value \\
\hline $\mathrm{C} 1 \mathrm{~S}$ & $\varepsilon 3 / \varepsilon 3$ & IGR & 0.11 & 0.13 & 0.24 & $2.5 \times 10^{-3}$ & 0.30 & $2.0 \times 10^{-4}$ \\
\hline $\mathrm{C} 2$ & $\varepsilon 3 / \varepsilon 3$ & $\mathrm{AR}$ & 0.05 & 0.53 & 0.17 & 0.04 & 0.30 & $2.2 \times 10^{-4}$ \\
\hline CD1D & $\varepsilon 3 / \varepsilon 3$ & $\mathrm{AR}$ & 0.28 & $3.4 \times 10^{-3}$ & 0.21 & 0.05 & 0.46 & $1.7 \times 10^{-5}$ \\
\hline CD38 & $\varepsilon 3 / \varepsilon 4$ & IGR & 0.09 & 0.25 & 0.07 & 0.41 & 0.39 & $6.1 \times 10^{-6}$ \\
\hline CFB & $\varepsilon 3 / \varepsilon 3$ & IGR & 0.16 & 0.05 & 0.46 & $2.2 \times 10^{-7}$ & 0.26 & $5.4 \times 10^{-3}$ \\
\hline DHX58 & $\varepsilon 3 / \varepsilon 3$ & IGR & 0.11 & 0.34 & 0.20 & 0.13 & 0.51 & $7.1 \times 10^{-5}$ \\
\hline ELF4 & $\varepsilon 3 / \varepsilon 4$ & $\mathrm{AR}$ & 0.32 & $8.7 \times 10^{-4}$ & 0.46 & $1.9 \times 10^{-5}$ & 0.26 & 0.02 \\
\hline FOSL1 & $\varepsilon 3 / \varepsilon 4$ & TSN & 0.20 & $3.1 \times 10^{-4}$ & 0.18 & $3.1 \times 10^{-3}$ & 0.08 & 0.21 \\
\hline HLA-A & $\varepsilon 3 / \varepsilon 3$ & AR; IGR & 0.27 & 0.03 & 0.38 & $3.9 \times 10^{-3}$ & 0.53 & $7.0 \times 10^{-5}$ \\
\hline IDH2 & $\varepsilon 3 / \varepsilon 3$ & $\mathrm{P}$ & 0.09 & 0.43 & 0.24 & 0.05 & 0.61 & $3.6 \times 10^{-7}$ \\
\hline IER3 & $\varepsilon 3 / \varepsilon 4$ & TSN & 0.14 & 0.06 & 0.37 & $3.5 \times 10^{-6}$ & 0.07 & 0.43 \\
\hline IFITM2 & $\varepsilon 3 / \varepsilon 3$ & IGR & 0.19 & 0.01 & 0.48 & $2.9 \times 10^{-9}$ & 0.18 & 0.03 \\
\hline IRF7 & $\varepsilon 3 / \varepsilon 4$ & AR; IGR & 0.24 & $9.5 \times 10^{-4}$ & 0.41 & $2.8 \times 10^{-7}$ & 0.20 & 0.02 \\
\hline ISG20 & $\varepsilon 3 / \varepsilon 4$ & IGR & 0.21 & $3.6 \times 10^{-3}$ & 0.34 & $1.9 \times 10^{-5}$ & 0.29 & $4.4 \times 10^{-4}$ \\
\hline LITAF & $\varepsilon 3 / \varepsilon 4$ & TSN & 0.27 & $4.1 \times 10^{-3}$ & 0.39 & $2.1 \times 10^{-4}$ & 0.43 & $3.9 \times 10^{-5}$ \\
\hline MVP & $\varepsilon 3 / \varepsilon 3$ & IGR; P & 0.21 & 0.01 & 0.42 & $7.0 \times 10^{-6}$ & 0.45 & $2.4 \times 10^{-6}$ \\
\hline PFKFB3 & $\varepsilon 3 / \varepsilon 4$ & TSN & 0.30 & $5.6 \times 10^{-4}$ & 0.30 & $2.6 \times 10^{-3}$ & 0.35 & $4.0 \times 10^{-4}$ \\
\hline PML & $\varepsilon 3 / \varepsilon 4$ & IGR & 0.28 & 0.02 & 0.47 & $3.8 \times 10^{-4}$ & 0.50 & $2.0 \times 10^{-4}$ \\
\hline SERPING1 & $\varepsilon 3 / \varepsilon 3$ & IGR & 0.17 & 0.13 & 0.35 & $4.4 \times 10^{-3}$ & 0.50 & $4.7 \times 10^{-5}$ \\
\hline TNIP2 & $\varepsilon 3 / \varepsilon 4$ & TSN & 0.36 & $1.9 \times 10^{-3}$ & 0.59 & $4.7 \times 10^{-6}$ & 0.19 & 0.15 \\
\hline TRIP10 & $\varepsilon 3 / \varepsilon 4$ & TSN & 0.32 & $2.1 \times 10^{-4}$ & 0.35 & $3.4 \times 10^{-4}$ & 0.06 & 0.58 \\
\hline
\end{tabular}

I-CAM: Intercellular Adhesion Molecule 1; SAA: Serum Amyloid A; V-CAM1: Vascular Cell Adhesion Molecule 1; AR: Allograft rejection; IGR: Interferon gamma response; P: Peroxisome; TSN: Tnfa signaling via nfkb

Results in bold surpass the multiple-testing threshold $\left(\mathrm{P}<4.67 \times 10^{-4}\right)$. 


\section{Figure 1}
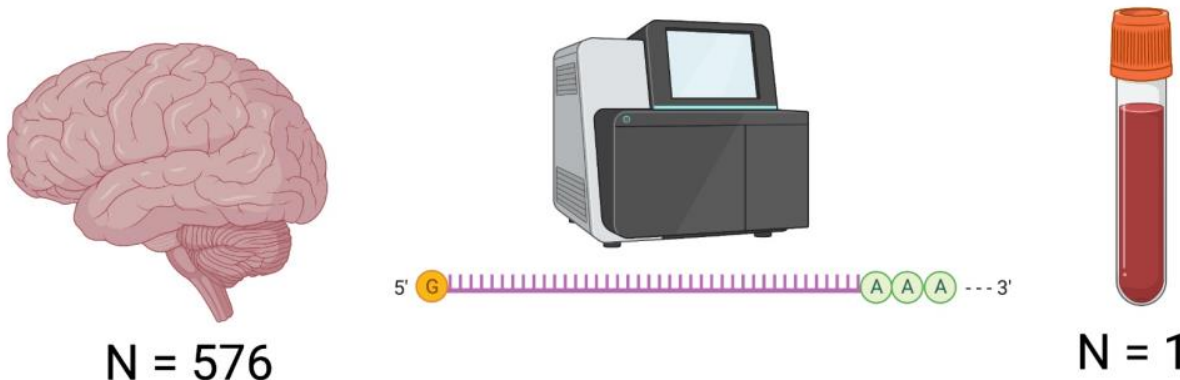

$$
N=576
$$

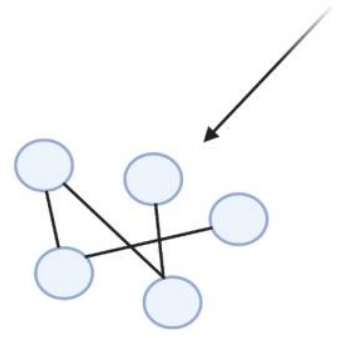

Gene Co-expression Networks in Brain Reproduced in Blood

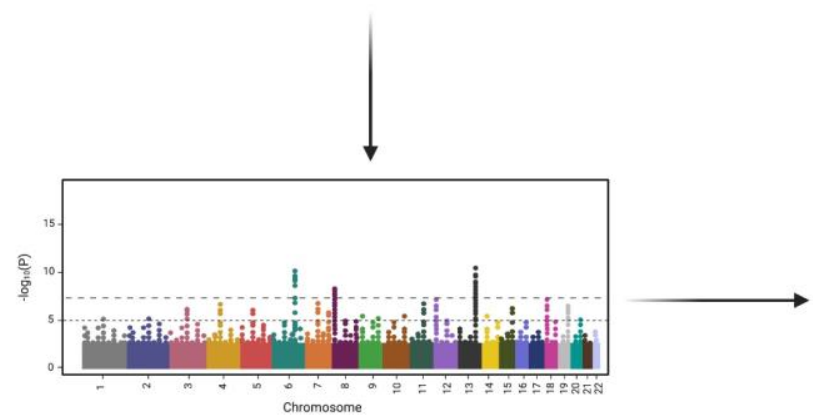

GWAS Gene Enrichment (AD,

Braak Stage, Plaque Score)

\section{$\mathrm{N}=179$}

Statify by $A P O E$ genotype ( $22 / \varepsilon 3, \varepsilon 3 / \varepsilon 3, \varepsilon 3 / \varepsilon 4$, total sample)

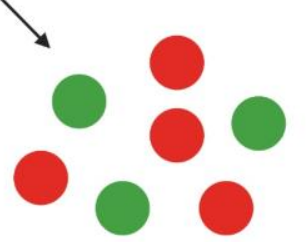

AD Differentially Expressed Genes in Blood and Brain

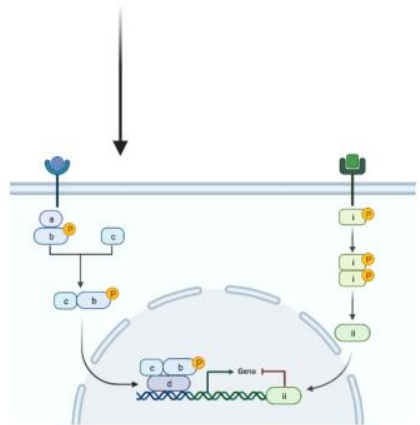

Pathway Analysis

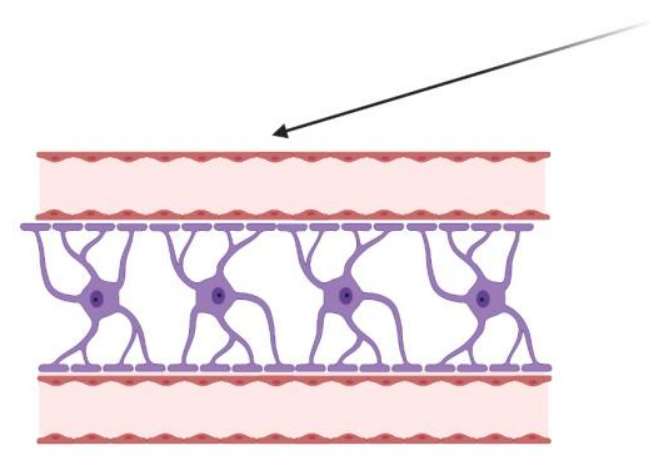

Association of Expression of Pathway Genes with Vascular Damage Proteins 
Figure 2
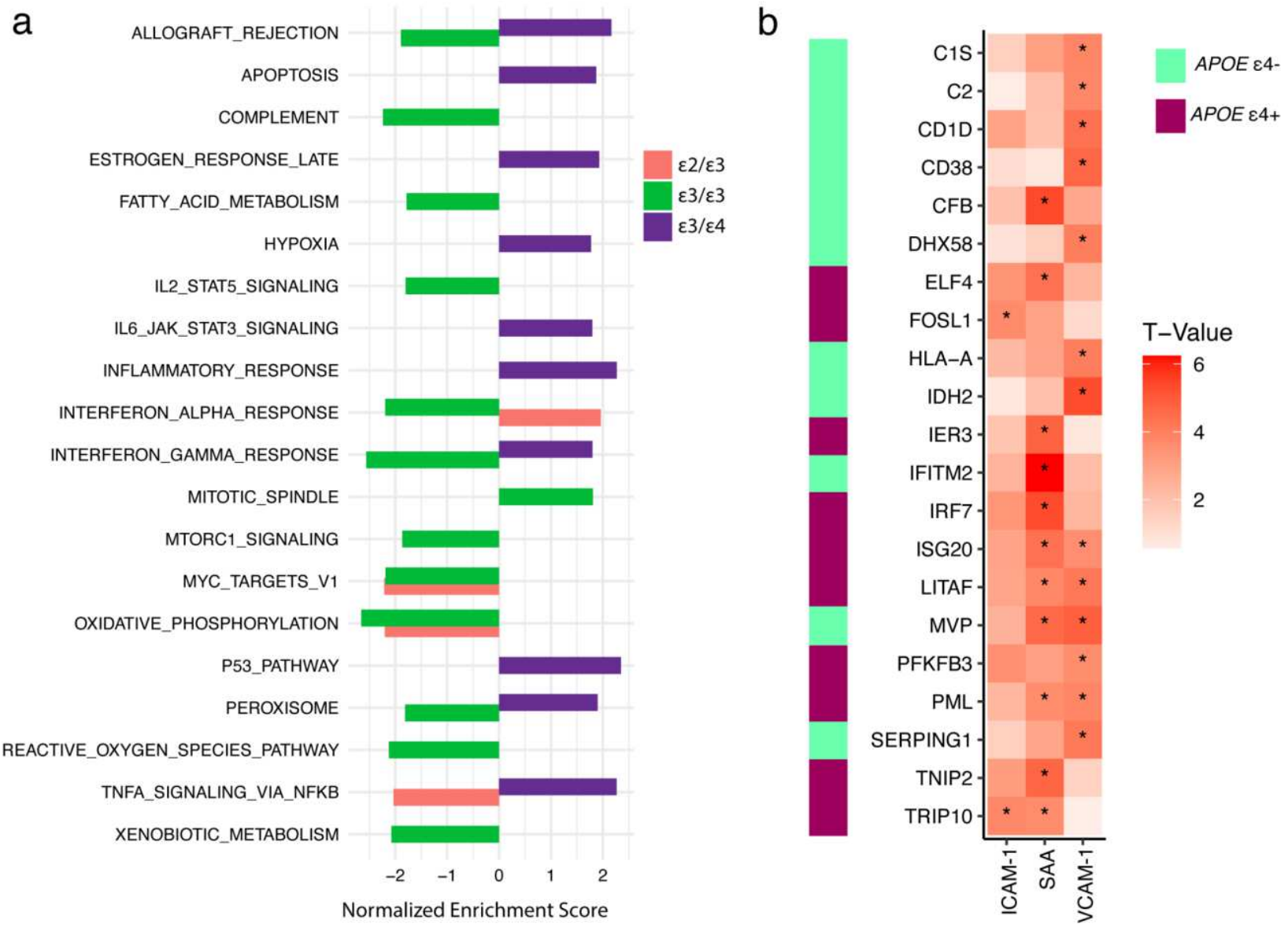


\section{Figure 3}

a

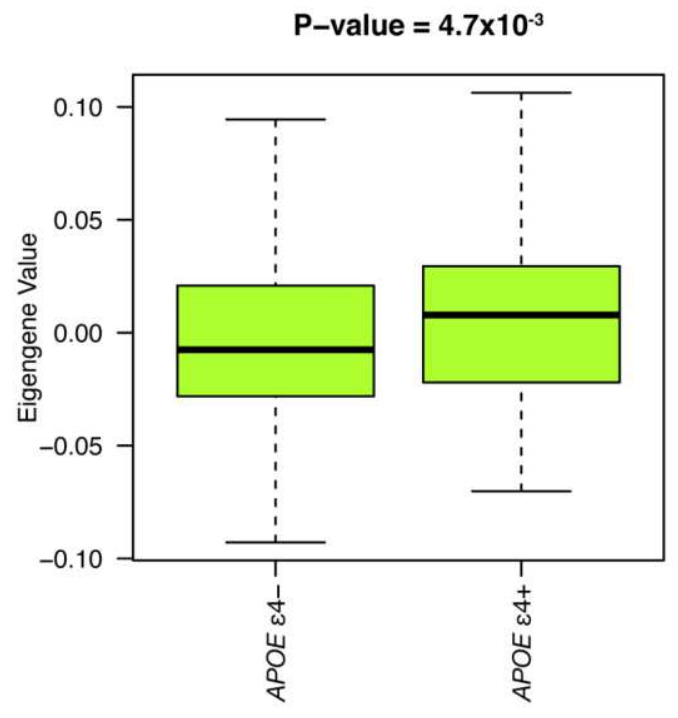

C

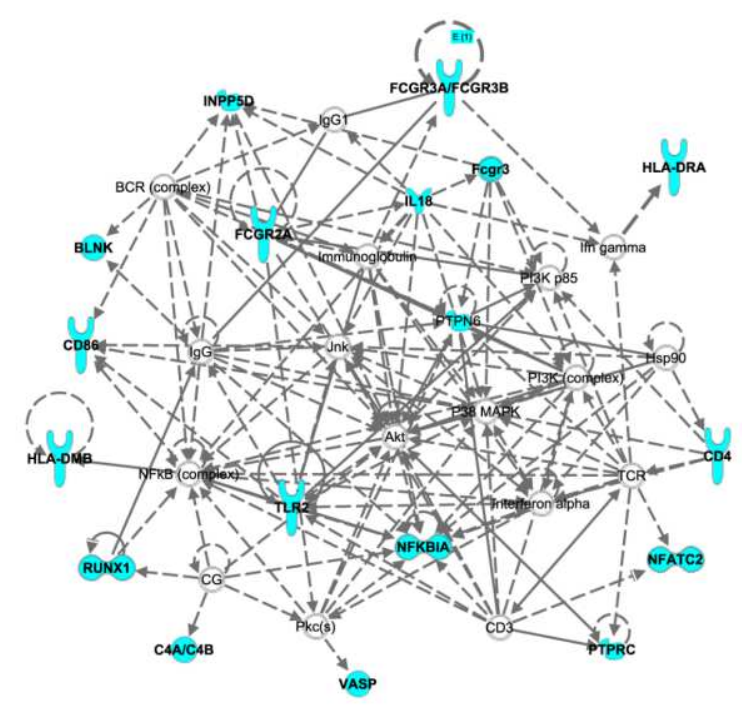

b

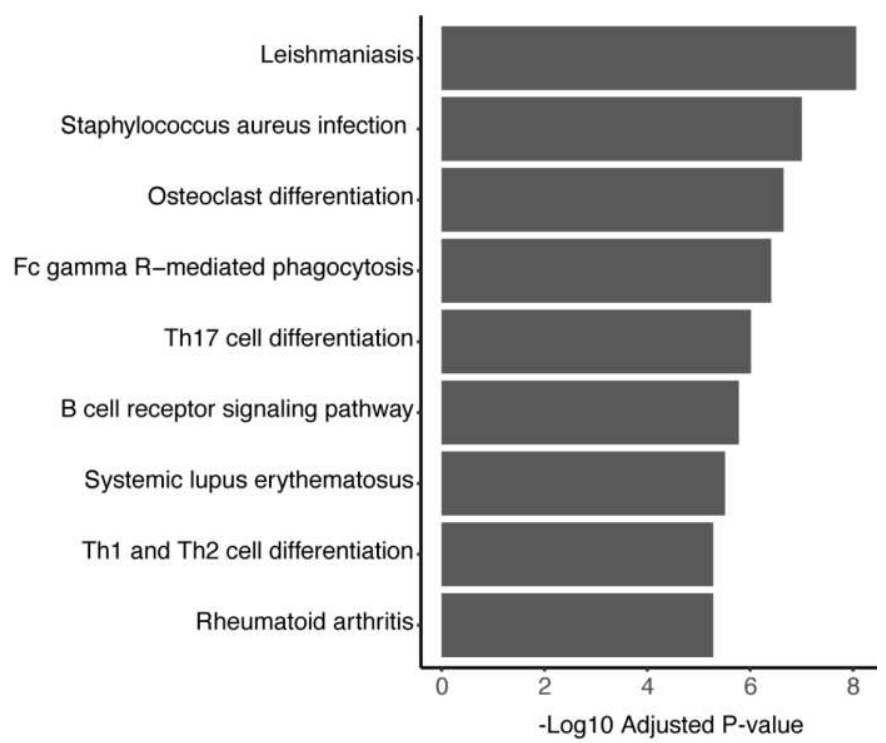

d

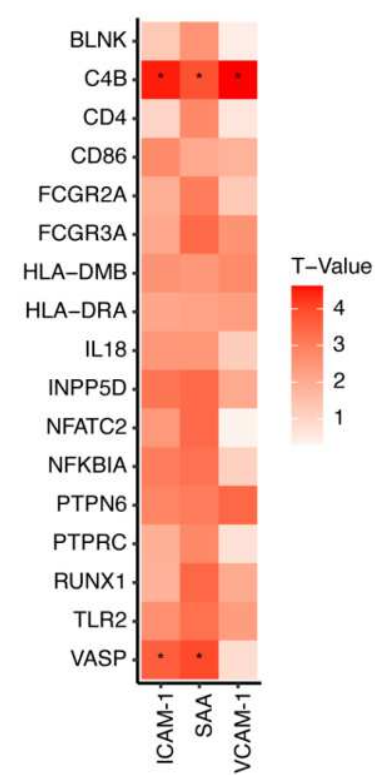




\section{Supplementary Files}

This is a list of supplementary files associated with this preprint. Click to download.

- PanitchSupplementarylnformation.pdf 